\title{
The Barrel TOF detector for PANDA
}

D. Steinschaden ${ }^{* 1}, K$. Dutta $^{2}$, L. Gruber ${ }^{1}$, K. Suzuki $^{1}$, M. Böhm ${ }^{3}$, M. Cardinali ${ }^{4}$, M. Hoek $^{4}$, K. Kalita ${ }^{2}$, K. Götzen ${ }^{6}$, H. Kumawat ${ }^{5}$, W. Lauth ${ }^{4}$, A. Lehmann ${ }^{3}$, J. Marton ${ }^{1}$, H. Orth $^{6}$, A. Parmar ${ }^{5}$, B. J. Roy ${ }^{5}$, C. Sonika ${ }^{5}$, L. Schmitt ${ }^{7}$, C. Schwarz ${ }^{6}$, C. Sfienti ${ }^{4}$, M. Thiel $^{4}$

${ }^{1}$ Stefan Meyer Institute for Subatomic Physics, Austrian Academy of Sciences, Vienna, Austria

${ }^{2}$ Department of Physics, Gauhati Universitiy, Guwahati, India

${ }^{3}$ Friedrich Alexander-University of Erlangen-Nuremberg, Erlangen, Germany

${ }^{4}$ Institut für Kernphysik, Johannes Gutenberg-University Mainz, Mainz, Germany

${ }^{5}$ Nuclear Physics Division, Bhabha Atomic Research Centre, Mumbai, India

${ }^{6}$ GSI Helholtzzentrum für Schwerionenforschung GmbH, Darmstadt, Germany

${ }^{7}$ Facility for Antiproton and Ion Research in Europe GmbH, Darmstadt, Germany

E-mail: dominik.steinschadendoeaw.ac.at

The PANDA experiment addresses fundamental questions in hadron and nuclear physics via interactions of antiprotons with protons. The experiment is currently under construction at the Facility for Antiproton and Ion Research (FAIR) in Darmstadt, Germany. The High Energy Storage Ring (HESR) will provide an antiproton beam with a momentum range of $1.5-15 \mathrm{GeV} / \mathrm{c}$ and an maximum collision rate on the fixed target of $20 \mathrm{MHz}$ is envisaged. The barrel-shaped time-of-flight (TOF) detector is planned as a scintillator tile hodoscope covering the central region of the PANDA detector and plays a crucial role in determining the time origin of the track. An online data reduction of about a factor 1000 is necessary and therefore the timing information of the scintillator tile hodoscope will be one of the key components. The detector provides particle identification also for slow particles below the momentum threshold of the DIRC. In order to fulfill the requirements, plastic scintillator tiles with minimum material budget read out by Silicon Photomultipliers (SiPMs) have been selected and the time resolution will be $\sigma<100 \mathrm{ps}$.

The European Physical Society Conference on High Energy Physics

22-29 July 2015

Vienna, Austria

* Speaker. 


\section{Introduction}

The PANDA experiment will be one of the major experiments installed at the Facility for Antiproton and Ion Research (FAIR) [1, 2], which is currently under construction as an expansion of the GSI Helmholz Centre (Darmstadt, Germany) and will be one of the largest accelerator facilities in the world. PANDA will be located at the High Energy Storage Ring (HESR), which will deliver a cooled antiproton beam in the momentum range of $1.5 \mathrm{GeV} / \mathrm{c}$ up to $15 \mathrm{GeV} / \mathrm{c}$ with a high relative beam momentum resolution of $10^{-5}$. At the interaction point with a fixed target a luminosity up to $2 \times 10^{32} \mathrm{~cm}^{-2} \mathrm{~s}^{-1}$ is reached. The experiment is focused on hadron physics in the charm region addressing: Hadron Spectroscopy, Nucleon Structure, Hadrons in Nuclear Medium, Hypernuclear Physics and Exotic Hadrons [3]. The PANDA detector is composed of two parts, the barrel shaped target spectrometer covering the interaction point and the forward spectrometer for small polar angles (see Fig. 1). The identification of charged particles is done by a combination of the tracking system, a Cherenkov detector and a fast timing detecor.

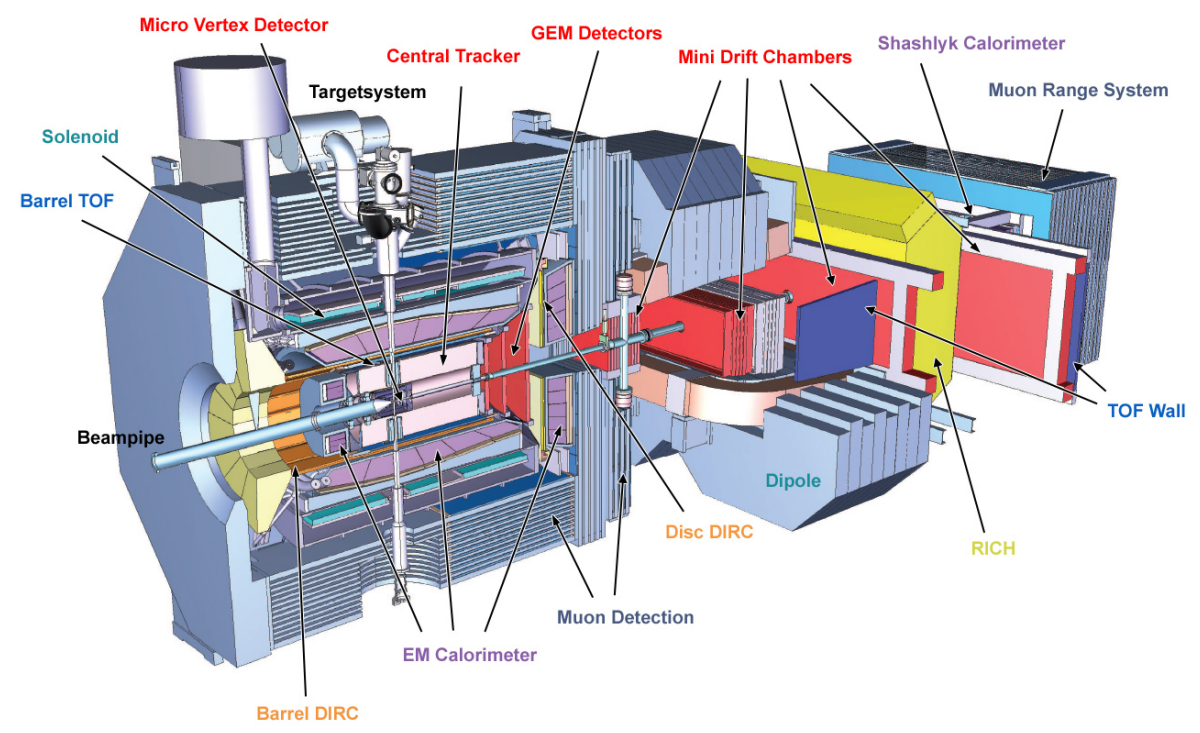

Figure 1: The PANDA spectrometer. The beam enters from the left and interacts in the target spectrometer.

\section{The Barrel time-of-flight detector}

The barrel time-of-flight (TOF) detector is motivated in terms of physics as well as technical benefits [4]. The TOF detector provides particle identification for charged particles and particularly complements the PID for particles below the momentum threshold of the barrel DIRC detector below $700 \mathrm{MeV} / \mathrm{c}$. Due to the triggerless continuous readout a software trigger, based on a detector like the scintillator based TOF detector, is needed to handle the event processing at the full interaction rate. In addition the time-of-flight detector delivers important timing information for any event building algorithm to avoid an event-mixing at high collision rates (see Fig. 2). PANDA will not have a start time detector for time-of-flight. Nevertheless using the TOF detector for relative timeof-flight in events where more than one track can be measured enables to calculate the time origin 

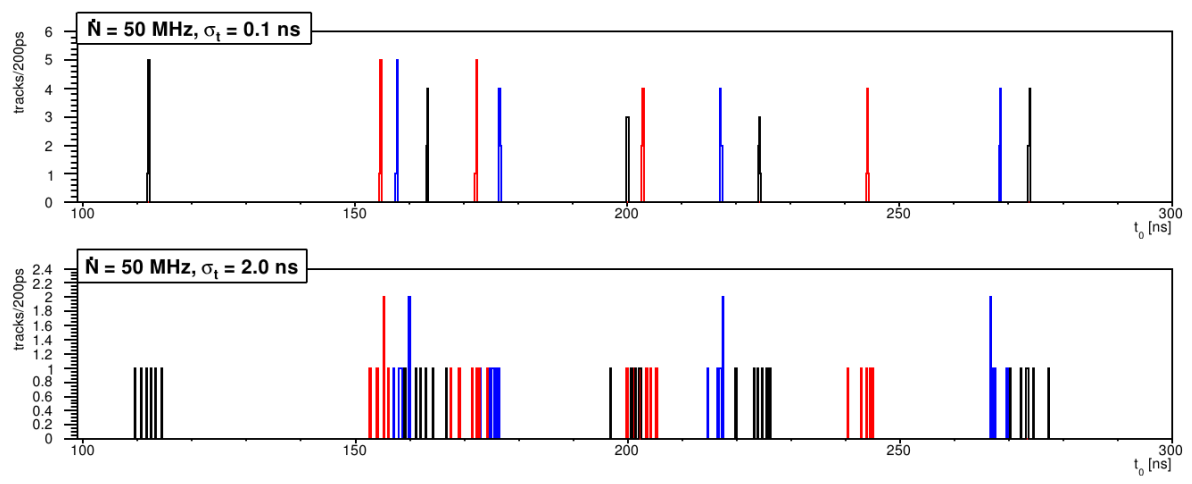

Figure 2: Illustration of $t_{0}$ distribution of reactions with 6 tracks at an instantaneous rate of $\mathrm{N}=50 \mathrm{MHz}$ and total time resolution of $\sigma=100 \mathrm{ps}$ (top) and $\sigma=2 \mathrm{~ns}$ (bottom). The three colors black, red and blue are cyclically used to mark time signals $t_{0}$ from different events [6].

of these tracks as described in [5]. The TOF detector also provides input for other systems and algorithms like additional track position information in beam direction for the pattern recognition, which may not be available from the inner tracking system. This subdetector sensitive to charged particles right in front of the electromagnetic calorimeter can also serve to detect electromagnetic preshowers starting inside the inner detectors. The strict requirements on the implementation of the TOF system demand optimization of material and thickness. The timing detector must get along with a minimum material budget below $2 \%$ of a radiation length and less than $2 \mathrm{~cm}$ radial thickness, including the readout electronics and mechanics and provide a large angular acceptance of $22^{\circ} \leq \theta \leq 140^{\circ}$. For the reasons given above a good time resolution $\sigma<100 \mathrm{ps}$ and a fast readout and signal processing is mandatory.

\section{Detector layout and optimization}

In order to reach the required time resolution of $\sigma<100 \mathrm{ps,} \mathrm{research} \mathrm{and} \mathrm{development} \mathrm{on}$ the detector design is needed. In the first design the TOF detector was planned as a Scintillator Tile Hodoscope (SciTil) composed out of 5760 small plastic scintillator tiles with sizes of about $30 \times 30 \times 5 \mathrm{~mm}^{3}$, read out by Silicon Photomultipliers (SiPMs). The achievable time resolution is influenced by several factors, namely the plastic scintillator, the photodetector and the readout electronics. The optimization has been done by performing laboratory tests using radioactive sources and lasers, test beam experiments and Monte Carlo simulations. Fig. 3 shows the single photon time resolution measured with a pulsed laser for a KETEK SiPM. Based on first optimization studies a SciTil prototype consisting of a $30 \times 30 \times 5 \mathrm{~mm}^{3}$ scintillator readout by $2 \mathrm{SiPMs}$ has been built and tested in a proton beam at the JESSICA beam line at the Forschungszentrum Jülich. The best time resolution of $\sigma=82.5 \pm 1.7$ ps has been obtained with KETEK SiPMs [6] (see Fig. 4).

The current design foresees in total 1920 larger plastic scintillator tiles with sizes of about $30 \times$ $90 \times 5 \mathrm{~mm}^{3}$. The scintillators are readout with $2 \times 4$ directly attached SiPMs in serial connection (see Fig. 5) and make use of the larger sensitive area to increase the number of detected photons to achieve a better time resolution as reported in Ref. $[9,10]$. In addition to the expected improvement in time resolution the number of readout channels is reduced due to the serial connection of 4 


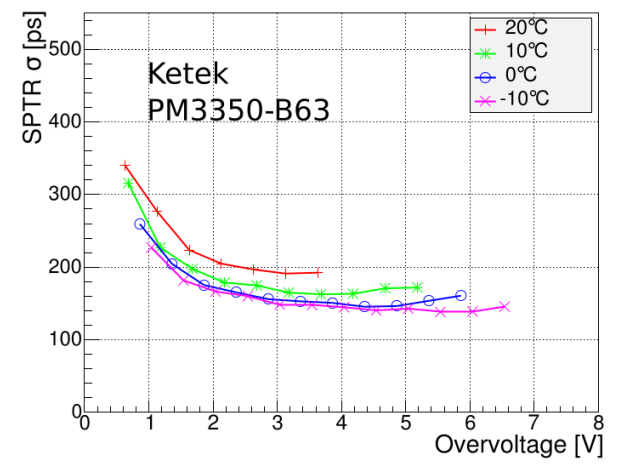

Figure 3: Single photon time resolution of a KETEK SiPM measured with a pulsed laser [7].

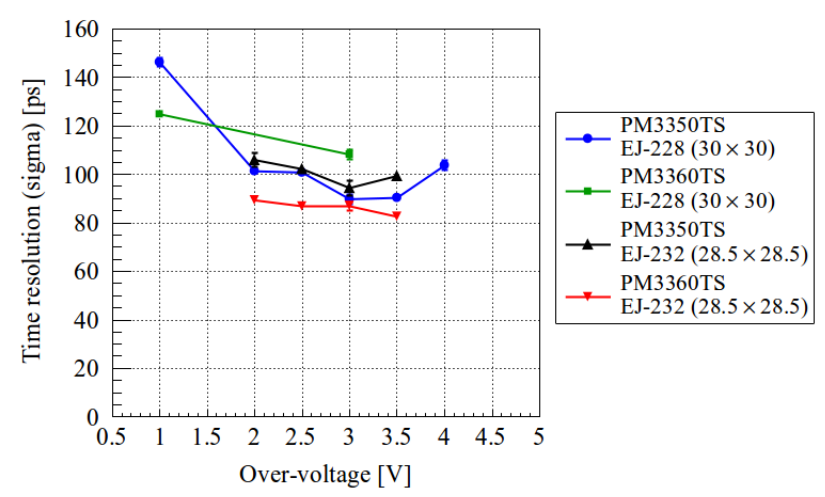

Figure 4: Measured time resolution with KETEK SiPMs with different scintillator materials in a proton beam [8].

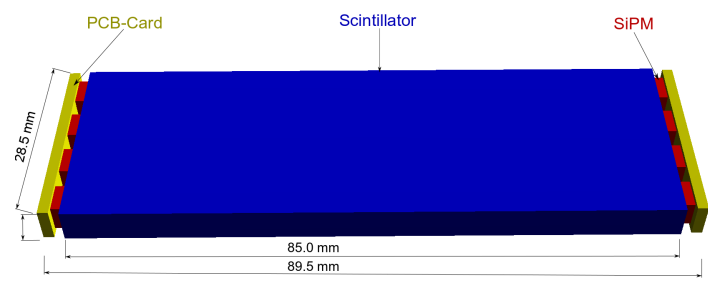

Figure 5: A detector module consisting of a scintillator tile (blue), readout by 4 SiPMs (red) on both sides.

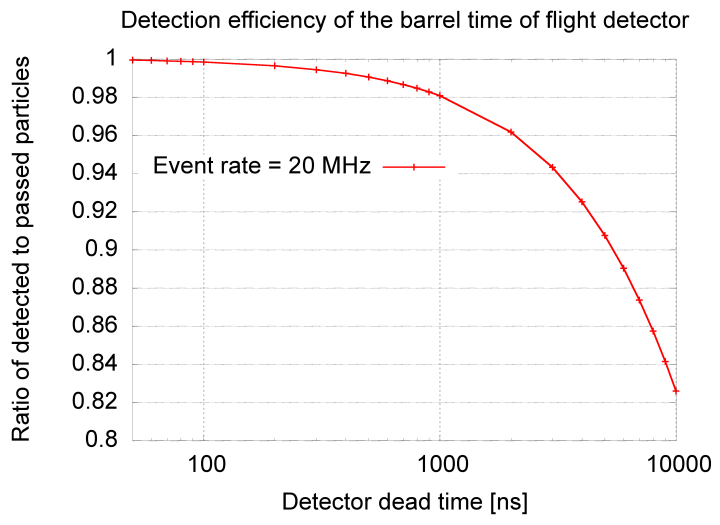

Figure 6: The ratio of detected to total passed particles in the TOF detector using $30 \times 90 \times 5 \mathrm{~mm}^{3}$ scintillating tiles as a function of the single module dead time is plotted. This calculated values only take primary particles into account.

SiPMs per side and a slightly better position resolution due to the utilization of the improved time difference resolution can be achieved.

For a first study for future comparision of the influence of different geometries as well as the benefit of the barrel TOF detector on the whole PANDA experiment an estimation of the detection efficiency as a function of the single detector module dead time of the SciTil has been calculated analytically. The calculation assumes in average an emission of 2 primary particles per event isotropically distributed in $20^{\circ} \leq \theta \leq 50^{\circ}$. The geometrical fill factor influenced by the dead area between the scintillating tiles needed for the SiPMs, readout boards and the wrapping of the scintillators is not taken into account. Fig. 6 shows the results for the average and the maximum expected event rate of the PANDA experiment and demonstrates the strong influence of the module dead time on the performance. 


\section{Conclusion and outlook}

It has been demonstrated experimentally that $\sigma<100$ ps for the TOF detector is achieved with the proposed detector layout. A new geometry is now under consideration and is expected to improve the performance of the SciTil. The effects of the changed geometry are still under investigation using Monte Carlo studies and laboratory tests to prove that the envisaged time and position resolution as well as the material requirements of the whole detector can be reached. Future Monte Carlo simulations will study in detail the influence of secondary and back scattered particles on the efficiency of the TOF detector as well as the benefits of the SciTil detector like the electromagnetic preshower detection on other sub-detector systems and tracking algorithms. Special effort is also put on the $R \& D$ of read out electronics. Therefore the simulations will provide input like the estimated dose distribution. 


\section{References}

[1] P.Spiller and G. Franchetti, The FAIR accelerator project at GSI, Nucl. Instrum. Meth. A 561 (2006) 305

[2] W.F. Henning, FAIR and its experimental programm, J. Phys. G 34 (2007) S551

[3] PANDA Collaboration, Physics Performance Report for PANDA:Strong Interactions Studies with Antiprotons, arXiv:0903.3905[hep-ex], Mar. 2009

[4] K. Goetzen et al., Proposal for a Scintillator Tile Hodoscope for PANDA, PANDA Note, Version 1.1, Mar. 2011

[5] A. gillitzer et al., Motivation of the Barrel Time-of-Flight Detector for PANDA, PANDA Note, Jan. 2011

[6] K. Goetzen, Influence of Particle Timing on Event Building, PANDA Note, Mar. 2011

[7] S. E. Brunner et al., Time resolution below 100 ps for SciTil detector of PANDA employing SiPM, JINST 9 (2014) C03010

[8] L. Gruber, Studies of SiPM photosensors for time-of-fight detectors within PANDA at FAIR, PhD Thesis (2014)

[9] A. Baldini et al., MEG Upgrade proposal, arXiv:1301.7225[physics.ins-det]

[10] P.W. Cattaneo et al., Development of High Precision Timing Counter Based on Plastic Scintillator with SiPM Readout, IEEE Trans. Nucl. Sci.61, 2657-2666 (2014) 